\title{
Breastfeeding Greater Than 6 Months Is Associated with Smaller Maternal Waist Circumference Up to One Decade After Delivery
}

\author{
Gabrielle G. Snyder, MPH, ${ }^{1}$ Claudia Holzman, DVM, MPH, PhD, ${ }^{2}$ Tao Sun, MS, ${ }^{3}$ \\ Bertha Bullen, $\mathrm{PhD},{ }^{2}$ Marnie Bertolet, $\mathrm{PhD}^{1,3}$ and Janet M. Catov, $\mathrm{PhD}^{1}$
}

\begin{abstract}
Background: Abdominal obesity is an important indicator of cardiometabolic dysfunction in later life. Prior studies have observed an inverse association between breastfeeding and maternal waist circumference (WC) in the years after pregnancy, but this may be due to bias resulting from systematic differences in women who do and do not breastfeed.

Materials and Methods: A total of 678 women enrolled in the Pregnancy Outcomes and Community Health (POUCH) cohort also participated in the POUCHmoms Study 7-15 years after delivery. Multivariable linear regression models and propensity scores were used to assess the relationship between WC measured at followup and self-reported history of breastfeeding duration of $>6$ months versus $\leq 6$ months.

Results: After a mean follow-up period of 11.0 (standard deviation =1.4) years, breastfeeding was significantly associated with smaller WC. A threshold effect was detected for women who reported breastfeeding $>6$ months; their adjusted mean WC was $3.5 \mathrm{~cm}$ (95\% confidence interval [CI]: -5.7 to -1.2$)$ smaller compared with women who breastfed $\leq 6$ months. The use of two propensity score approaches, weighted and matched, produced similar results; adjusted mean WC difference was $-3.6 \mathrm{~cm}$ (95\% CI: -5.6 to -1.6$)$ and $-3.1 \mathrm{~cm}$ (95\% CI: -5.5 to -0.7$)$, respectively. Conclusions: This study extends conventional observational study methods to incorporate propensity score approaches that make it possible to separate the study design from the study analysis to account for systematic differences in women who did and did not breastfeed. After reducing potential bias, breastfeeding for greater than 6 months was independently associated with smaller WC in the decade after delivery.
\end{abstract}

Keywords: breastfeeding, maternal obesity, waist circumference, propensity score

\section{Introduction}

T he World Health Organization (WhO) recommends that exclusive breastfeeding for 6 months is optimal for infant health. ${ }^{1}$ However, an equivalently optimal duration for mothers to experience health benefits from breastfeeding has yet to be determined. This may be due to conflicting evidence regarding the association between breastfeeding and maternal health outcomes in the years after pregnancy. ${ }^{2}$

Normal pregnancy is accompanied by weight gain and retention postpartum, and may contribute to increased insulin resistance and cardiovascular disease risk in later life. ${ }^{3-5}$ In particular, metabolically active visceral adipose tissue, a mea- sure of abdominal obesity primarily distributed about the intraabdominal organs, may increase substantially with a single pregnancy, independent of total body fat. ${ }^{6}$ Although body mass index (BMI) is commonly used in clinical and research practices to quantify overall adiposity, it does not distinguish among types of body fat mass or body fat distribution. ${ }^{7,8}$ Waist circumference (WC) as a measure of central adiposity has been shown to be a superior proxy for assessing long-term risk of coronary artery disease mortality, hypertension, diabetes, dyslipidemia, and the metabolic syndrome, independent of BMI. ${ }^{8-10}$

Postpartum milk production for breastfeeding requires mothers to expend nearly 500 additional kilocalories per day. ${ }^{11}$ Some, but not all, previous studies examining the association between breastfeeding and maternal health have found that

${ }^{1}$ Department of Epidemiology, Graduate School of Public Health, University of Pittsburgh, Pittsburgh, Pennsylvania.

${ }^{2}$ Department of Epidemiology and Biostatistics, College of Human Medicine, Michigan State University, East Lansing, Michigan.

${ }^{3}$ Department of Biostatistics, Graduate School of Public Health, University of Pittsburgh, Pittsburgh, Pennsylvania. 
longer breastfeeding duration may be related to promotion of healthy weight loss and body composition. ${ }^{12-14}$ However, this detected effect may depend on whether or not breastfeeding is exclusive. ${ }^{15}$ In methodologically rigorous studies, longer breastfeeding duration was most consistently related to less weight retention and abdominal visceral adiposity and smaller WC. ${ }^{16,17}$ However, a linear dose-response relationship between breastfeeding duration and maternal weight change has not been consistently observed. ${ }^{18}$

Prepregnancy obesity and healthy lifestyle continue to obfuscate associations between breastfeeding and maternal postpartum adiposity. ${ }^{19}$ Women who breastfeed may be systematically different from women who do not, in which the former are more likely to exhibit health-promoting behaviors. ${ }^{20}$ Propensity score analyses are one way to account for the potential bias that these systematic differences may introduce. For our purposes, the propensity score is the probability of breastfeeding $>6$ months and is estimated using logistic regression. Covariates in the propensity score model consist of variables before breastfeeding and are included to get the best estimate of the probability of breastfeeding $>6$ months. These propensity scores can be used analytically in two ways. First, women who breastfed $>6$ months are matched with women who breastfed $\leq 6$ months based on their propensity to breastfeed. Women with the same probability (one who breastfed and one who did not) are matched. Second, propensity scores can be used to create inverse probability score weights. This creates a pseudo-population in such a way that the groups are balanced based on the underlying probability of breastfeeding. ${ }^{21}$ Both approaches balance the baseline characteristics of women who breastfed for longer durations with women who breastfed for shorter durations or not at all. ${ }^{22}$ These are two robust methods that can be used to mitigate confounding when using observational study data to estimate treatment effect size. To our knowledge, propensity scores have not been applied to studies of breastfeeding and long-term maternal adiposity. ${ }^{23}$

Similar to WHO recommendations for infants, breastfeeding for 6 months may be optimal for mothers to experience long-term health benefits. We hypothesized that women who breastfed more than 6 months would have a smaller WC 7-15 years after delivery compared to women who breastfed less than or equal to 6 months, after accounting for prepregnancy and pregnancy factors that may influence the likelihood of breastfeeding. We used multivariable linear regression models as well as propensity score approaches to test this relationship.

\section{Materials and Methods}

\section{Subjects}

The Pregnancy Outcomes and Community Health (POUCH) Study enrolled 3019 pregnant women from 52 clinics in 5 Michigan communities (1998-2004) to investigate biomarkers of pathways to preterm delivery. English-speaking women at least 15 years of age with no history of diabetes and with a singleton pregnancy with no known fetal abnormalities were enrolled into the study cohort, oversampling African American women and women with unexplained high maternal serum alpha-fetoprotein (MSAFP). All POUCH Study participants completed in-depth in-person interviews and provided biologic samples at enrollment. A more detailed assessment was conducted in a subcohort of 1371 POUCH women, including placental histopathology, biomarker assays, and medical record abstraction. This subcohort included all women with preterm birth before 37 weeks of gestation, all women with unexplained high MSAFP (>2.0 multiples of the median), and a race/ethnicity stratified random sample of women with term delivery and normal MSAFP. ${ }^{24}$ Sampling weights were constructed to account for the cohort and subcohort sampling scheme.

At a follow-up visit, 7-15 years after the POUCH Study, 678 women returned as participants in the POUCHmoms Study (2010-2014), which was designed to examine the association between pregnancy factors and long-term maternal cardiovascular disease risk. Women who were pregnant or within 6 months postpregnancy were ineligible for the POUCHmoms Study. Postpregnancy data were collected at this follow-up visit through self-report and direct measure. For our analyses, we excluded 2 women with invalid WC measurements, leaving an analytic sample of 676 participants with complete outcome and exposure data (Fig. 1). Women from the POUCH subcohort who returned to participate were compared to those who did not return for the follow-up visit. The former were slightly older ( $>30$ years) and had higher levels of education. In addition, a greater percentage of women followed were White, had high MSAFP levels, and were not insured by Medicaid. The institutional review boards at Michigan State University and the University of Pittsburgh approved the POUCHmoms Study and the latter approved this analysis.

\section{Exposure}

Breastfeeding duration for the POUCH Study pregnancy was reported at the follow-up visit. Participants were asked if they breastfed (yes/no) and the age of the infant in months when they stopped nursing. If breastfeeding was intermittent, participants were asked to report infant age when all breastfeeding stopped. These questions did not differentiate between feeding at the breast and pumping. In linear regression models, breastfeeding was evaluated in three ways: (1) as a continuous variable; (2) categorized into four groups: none, $>0-3$ months, $>3-6$ months, and $>6$ months to facilitate comparison with prior studies; and (3) dichotomized by $\leq 6$ months and $>6$ months to compare to propensity score results. For propensity score modeling, which was estimated with a logistic regression, matching was done across two groups. Thus, breastfeeding duration was defined as a binary variable ( $\leq 6$ months and $>6$ months).

\section{Outcome}

An average of three WC measurements, collected by Gulick tape measure at the peak of expiration by trained research assistants, was used as a continuous measure of central adiposity. ${ }^{24}$ To explore a clinically meaningful measure of body composition, WC $\geq 88 \mathrm{~cm}$ was used to indicate central adiposity in women. ${ }^{25}$ In addition, weight and height were measured using standardized methods to assess BMI as a secondary outcome.

\section{Covariates}

Variables collected from the POUCH Study pregnancy medical records that were considered in this analysis included gestational weight gain (GWG) adequacy defined by the Institute of Medicine, hypertensive disorders of pregnancy (preeclampsia and gestational hypertension), prepregnancy chronic hypertension defined as blood pressure $\geq 140 \mathrm{mmHg}$ 


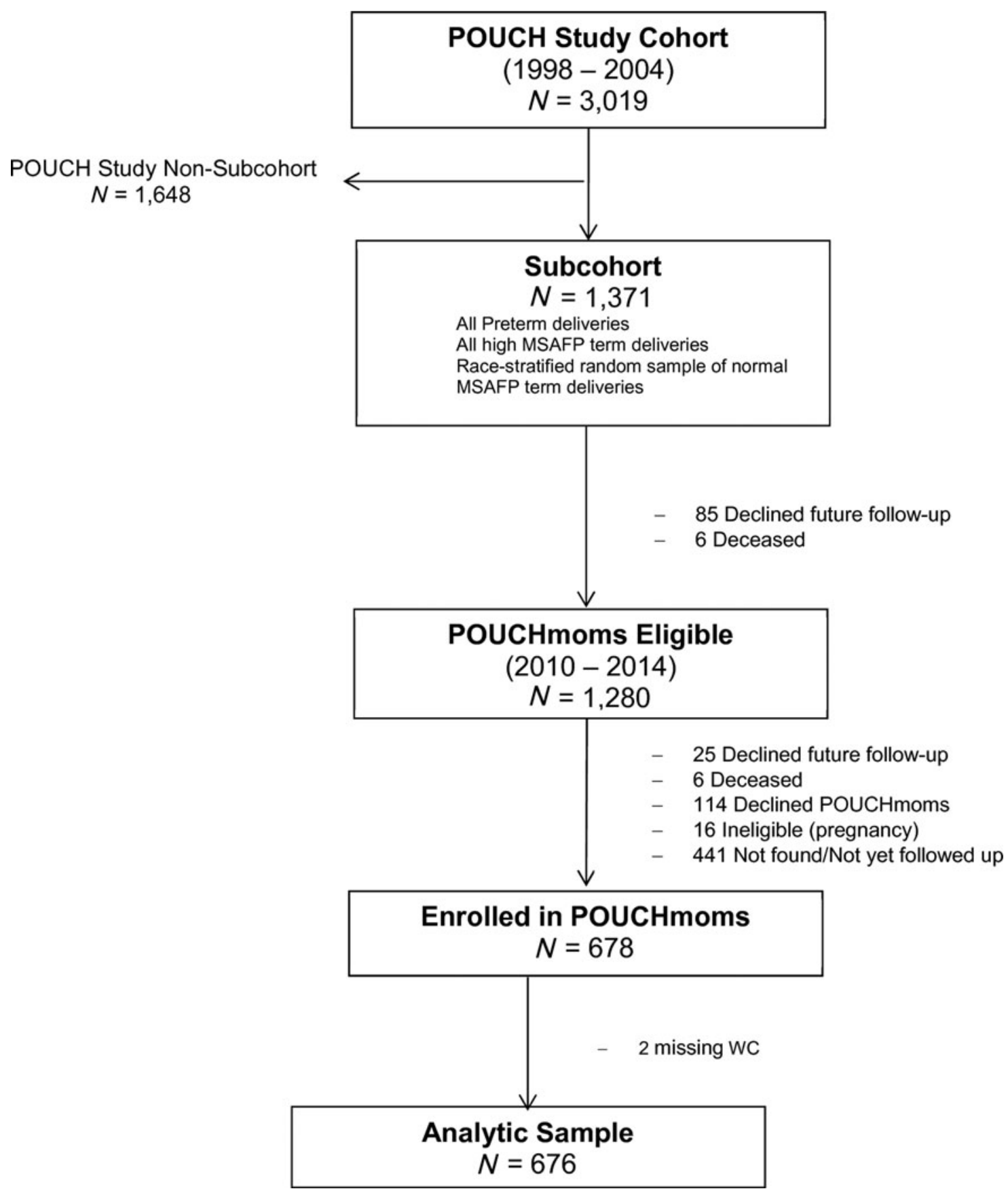

FIG. 1. Flow chart of participants in POUCHmoms Study, 2011-2014. POUCH, Pregnancy Outcomes and Community Health.

systolic or $90 \mathrm{mmHg}$ diastolic before pregnancy or before 20 weeks gestation without resolution postpartum, or using antihypertensive medications during this same period, preterm birth ( $<37$ weeks gestation), and gestational diabetes. ${ }^{26,27}$ Maternal race/ethnicity was collected by self-report at the time of POUCH enrollment. Prepregnancy BMI was computed based on self-reported weight at the POUCH Study in kilograms and divided by squared height in meters. At follow-up, maternal age, total number of live births, and educational attainment ( $<12$ years of formal education, high school diploma, vocational training/some college, and col- lege graduate) were assessed by a structured interview. Three resting seated measures of systolic and diastolic blood pressures were taken; the average of the second and third was used for our measure. A diet quality score was calculated from the Block Food Frequency Questionnaire, with a higher score indicating a higher quality diet. ${ }^{28}$ An average number of moderate-vigorous physical activity hours per week was self-reported by the Modified Activity Questionnaire (MAQ). ${ }^{29}$ Women were asked about smoking and whether they received Medicaid insurance coverage for the periods before, during, and/or after the POUCH Study pregnancy. 
Table 1. Maternal Characteristics During Pregnancy and 7-15 Years After Delivery AcCording to BreastfeEding Duration

\begin{tabular}{|c|c|c|c|c|}
\hline Characteristic & $\mathrm{N}$ & $\leq 6$ months $(\mathrm{N}=496)$ & $>6$ months $(\mathrm{N}=180)$ & $\mathrm{p}$ \\
\hline \multicolumn{5}{|l|}{ POUCH pregnancy (1998-2004) } \\
\hline Gestational age at enrollment, weeks & 676 & $22.2(0.12)$ & $22.5(0.20)$ & 0.323 \\
\hline Gestational age at delivery, weeks & 676 & $38.9(0.08)$ & $39.5(0.12)$ & $<0.001$ \\
\hline Maternal age at delivery, years & 676 & $25.8(0.28)$ & $29.5(0.46)$ & $<0.0001$ \\
\hline Prior parity & 676 & $0.9(0.05)$ & $0.9(0.11)$ & \multirow{7}{*}{$\begin{array}{l}0.001 \\
0.044\end{array}$} \\
\hline \multicolumn{4}{|l|}{ Prepregnancy BMI, $\mathrm{kg} / \mathrm{m}^{2}, n(\%)$} & \\
\hline Normal weight, $<25$ & 332 & $228(46.0)$ & $104(59.0)$ & \\
\hline Overweight, $25-29.9$ & 142 & $106(21.9)$ & $36(20.4)$ & \\
\hline Obese Class I, 30-34.9 & 98 & $76(13.1)$ & $22(11.8)$ & \\
\hline Obese Class II, 35-39.9 & 55 & $48(10.8)$ & $7(4.5)$ & \\
\hline Obese Class III, $\geq 40$ & 49 & $38(8.2)$ & $11(4.4)$ & \\
\hline \multicolumn{4}{|l|}{ GWG, $n(\%)$} & \multirow[t]{4}{*}{0.006} \\
\hline Inadequate & 112 & $95(18.8)$ & $17(8.4)$ & \\
\hline Adequate & 124 & $83(17.8)$ & $41(27.7)$ & \\
\hline Excessive & 390 & $283(63.4)$ & $107(64.0)$ & \\
\hline \multicolumn{4}{|l|}{ Hypertensive disorders, $n(\%)$} & \multirow[t]{4}{*}{0.014} \\
\hline None & 599 & $445(92.5)$ & $154(83.8)$ & \\
\hline Preeclampsia or gestational hypertension & 52 & $34(5.5)$ & $18(11.0)$ & \\
\hline Chronic hypertension & 25 & $17(2.0)$ & $8(5.2)$ & \\
\hline Gestational diabetes, $n(\%)$ & 40 & $24(5.2)$ & $16(9.8)$ & 0.080 \\
\hline Preterm birth, $n(\%)$ & 169 & $137(12.7)$ & $32(6.3)$ & $<0.001$ \\
\hline \multicolumn{4}{|l|}{ Race, $n(\%)$} & \multirow[t]{4}{*}{$<0.0001$} \\
\hline White & 390 & $263(63.2)$ & $127(78.5)$ & \\
\hline African American & 248 & $210(30.7)$ & $38(11.0)$ & \\
\hline Asian or Native American & 38 & $23(6.1)$ & $15(10.5)$ & \\
\hline \multicolumn{4}{|l|}{ Medicaid status, $n(\%)$} & \multirow[t]{2}{*}{$<0.0001$} \\
\hline On Medicaid & 346 & $296(56.5)$ & $50(21.7)$ & \\
\hline & 329 & $199(43.5)$ & $130(78.3)$ & \\
\hline \multicolumn{4}{|l|}{ Education, $n(\%)$} & \multirow[t]{5}{*}{$<0.0001$} \\
\hline$<12$ years & 130 & $121(22.6)$ & $9(5.5)$ & \\
\hline HS Diploma & 178 & $144(29.0)$ & $34(15.3)$ & \\
\hline Vocational training/Some college & 203 & 149 (31.4) & $54(30.4)$ & \\
\hline College graduate & 165 & $82(17.0)$ & $83(48.8)$ & \\
\hline \multicolumn{4}{|l|}{ Smoking, $n(\%)$} & \multirow[t]{5}{*}{$<0.0001$} \\
\hline Did not smoke during pregnancy & 491 & $337(67.1)$ & $154(85.5)$ & \\
\hline Stopped before enrollment & 67 & $50(10.2)$ & $17(10.5)$ & \\
\hline Smoked $<1 / 2$ pack/day at enrollment & 76 & $71(13.5)$ & $5(2.4)$ & \\
\hline Smoked $>1 / 2 \mathrm{pack} /$ day at enrollment & 42 & $38(9.2)$ & $4(1.6)$ & \\
\hline POUCHmoms follow-up visit $(2010-2014)$ & & & & \\
\hline Delivery to follow-up, years & 676 & $11.1(0.7)$ & $10.8(0.1)$ & 0.014 \\
\hline Systolic blood pressure, $\mathrm{mmHg}$ & 676 & $115.4(0.7)$ & $113.0(1.2)$ & 0.076 \\
\hline Diastolic blood pressure, $\mathrm{mmHg}$ & 676 & $76.4(0.6)$ & $73.5(1.0)$ & 0.011 \\
\hline Waist circumference, $\mathrm{cm}$ & 676 & $93.6(0.9)$ & $85.6(1.2)$ & $<0.0001$ \\
\hline Hip circumference, $\mathrm{cm}$ & 676 & $115.2(1.0)$ & $107.8(1.3)$ & $<0.0001$ \\
\hline Waist-hip ratio & 676 & $0.81(0.00)$ & $0.79(0.00)$ & 0.005 \\
\hline Diet quality score & 675 & $51.9(0.5)$ & $56.9(0.9)$ & $<0.0001$ \\
\hline MAQ average physical activity, hours/week & 676 & $3.8(0.3)$ & $5.1(0.5)$ & 0.018 \\
\hline BMI, $\mathrm{kg} / \mathrm{m}^{2}, n(\%)$ & & & & $<0.0001$ \\
\hline Normal weight, $<25$ & 162 & $102(20.6)$ & $60(35.1)$ & \\
\hline Overweight, $25-29.9$ & 159 & $104(21.4)$ & $55(32.4)$ & \\
\hline Obese Class I, 30-34.9 & 136 & $108(21.1)$ & $28(15.5)$ & \\
\hline Obese Class II, 35-39.9 & 95 & $76(15.4)$ & $19(8.4)$ & \\
\hline Obese Class III, $\geq 40$ & 124 & $106(21.5)$ & $18(8.6)$ & \\
\hline Total parity, $n(\%)$ & & & & 0.153 \\
\hline 1 & 60 & $47(8.7)$ & $13(6.2)$ & \\
\hline 2 & 259 & $178(37.8)$ & $81(49.1)$ & \\
\hline 3 & 208 & $155(32.8)$ & $53(27.2)$ & \\
\hline $4+$ & 149 & $116(20.7)$ & $33(17.5)$ & \\
\hline Education level, $n(\%)$ & & & & $<0.0001$ \\
\hline$<12$ years & 52 & $49(9.1)$ & $3(1.5)$ & \\
\hline HS Diploma & 94 & $84(16.1)$ & $10(5.3)$ & \\
\hline Vocational training/Some college & 339 & $258(53.9)$ & $81(41.4)$ & \\
\hline College graduate & 191 & $105(20.9)$ & $86(51.8)$ & \\
\hline
\end{tabular}


Table 1. (Continued)

\begin{tabular}{lrcrc}
\hline Characteristic & $\mathrm{N}$ & $\leq 6$ months $(\mathrm{N}=496)$ & $>6$ months $(\mathrm{N}=180)$ & $\mathrm{p}$ \\
\hline Cumulative Medicaid status, $n(\%)$ & & & & $<0.0001$ \\
Never on Medicaid & 290 & $169(38.7)$ & $121(74.7)$ & \\
Medicaid before POUCH only & 134 & $107(21.3)$ & $27(13.0)$ & \\
Medicaid after POUCH only & 39 & $30(4.8)$ & $9(3.7)$ & \\
Medicaid before, during, and after POUCH & 212 & $189(35.2)$ & $23(8.6)$ & $<0.0001$ \\
Cumulative smoking, $n$ (\%) & & & $125(70.0)$ & \\
Never smoked & 366 & $241(48.0)$ & $28(17.0)$ & \\
Former smoker & 93 & $65(12.4)$ & $12(4.8)$ & \\
Current, but not during POUCH pregnancy & 71 & $59(11.9)$ & $15(8.2)$ & \\
Current and during POUCH pregnancy & 145 & $130(27.7)$ & \\
\hline
\end{tabular}

Mean (SE) unless otherwise noted.

Accounting for sampling weights.

BMI, body mass index; GWG, gestational weight gain; MAQ, Modified Activity Questionnaire; POUCH, Pregnancy Outcomes and Community Health; SE, standard error.

\section{Statistical analysis}

Univariate associations of breastfeeding duration by prepregnancy risk factors and pregnancy outcomes, socioeconomic status, and healthy lifestyle factors were assessed using ANOVA (analysis of variance) and chi-square tests. All model assumptions were checked and no major violations were found. A series of four nested multivariable linear regression models, with each model adding a set of related factors, was used to estimate the association between breastfeeding duration and central adiposity. Following initial adjustment of continuous prepregnancy BMI to estimate $\mathrm{WC}$ at follow-up (Model 1), Model 2 incorporated maternal age, race/ethnicity, and factors related to child-bearing, including parity, GWG, and adverse pregnancy outcomes of preterm birth, gestational diabetes, and hypertensive disorders. Subsequent models included categorical variables for educational attainment and Medicaid insurance history as proxies for socioeconomic status (Model 3), and lifestyle factors of smoking history, diet quality, and physical activity (Model 4). Interaction terms were also tested to determine if GWG, smoking history, follow-up time, or parity may modify the association between breastfeeding duration and WC. Sampling weights were applied to calculate estimates reflective of the original source population.

Propensity score analyses were completed to mimic a randomized controlled trial comparing participants who breastfed $>6$ months (treatment) versus $\leq 6$ months (control). Propensity scores were created to determine the probability of breastfeeding $>6$ months using prepregnancy factors, and did not take the outcome (WC or BMI) into account. In addition, diagnostics for propensity score approaches, such as absolute standardized differences that assess overlap between treatment and control groups, were used to determine whether models have been adequately specified. ${ }^{22}$

We applied two propensity score approaches: (1) propensity matching and (2) propensity weighting. ${ }^{30}$ Although the former method can be used to directly compare women with similar propensity scores, who did and did not breastfeed, it reduces the total sample size by half. Therefore, we also used propensity weighting to utilize the entire study sample. First, logistic regression models were used to estimate the breastfeeding propensity scores based on self-reported prepregnancy and delivery covariates only, including self-reported weight, height, BMI, race/ethnicity, parity, age, gestational age in weeks, preterm birth, preeclampsia and gestational hypertension, smoking during pregnancy, GWG, gestational diabetes, education, and Medicaid status during pregnancy. Propensity matching created one-to-one matched pairs of treatment and control individuals who had similar propensity score values within a caliper width equal to 1.3 of the standard deviation. To draw valid inferences about the association of breastfeeding and WC, we used absolute standardized differences as a diagnostic tool to determine that measured baseline covariates for the breastfeeding groups ( $>6$ months vs. $\leq 6$ months) had similar distributions. ${ }^{22}$ In propensity score weighting, the same propensity score was used to determine each participant's inverse of probability treatment weighting. We then controlled for possible confounding by measures collected at follow-up by fitting propensity score-weighted linear regression models and adjusting for the same covariates as in the unweighted multivariable linear regression models.

We compared the multivariable linear regression results to the two propensity score methods to determine whether or not multivariable linear regression may adequately estimate the association between breastfeeding duration and WC. Analyses were performed in SAS (version 9.4; SAS Institute, Inc., Cary, NC) and R (version 3.3.0; R Foundation for Statistical Computing, Vienna, Austria), and assumed 95\% confidence intervals (CIs) with a significance level of 0.05 .

\section{Results}

Overall, $61.8 \%$ of the POUCHmoms participants reported having breastfed for some duration. Specifically, 38.2\% of the participants did not breastfeed following the POUCH pregnancy, while $22.0 \%, 13.2 \%$, and $26.6 \%$ breastfed for $>0$ 3 months, $>3-6$ months, and $>6$ months, respectively. There was no difference in mean WC between women who reported no breastfeeding, breastfeeding for $>0-3$ months, or $>3-6$ months $(95.2,91.5$, and $91.6 \mathrm{~cm}$, respectively, $p=0.08$ ). Further analyses combined all women breastfeeding $\leq 6$ months as the referent group.

In unadjusted analyses presented in Table 1, women who breastfed $>6$ months were older and delivered POUCH Study 
infants with greater gestational age $(p \leq 0.001)$ compared to women who breastfed $\leq 6$ months. They were also less likely to be obese before pregnancy $(p=0.044)$, have inadequate GWG $(p=0.006)$, or a preterm birth $(p<0.001)$, but were more likely to have chronic or pregnancy-related hyperten- sive disorders ( $p=0.014)$. Longer breastfeeding duration was also associated with higher educational attainment and lower rates of smoking during pregnancy and Medicaid insurance coverage $(p<0.0001)$. African American women breastfed for considerably shorter durations, comprising $11.0 \%$ of

Table 2. Maternal Characteristics During Pregnancy and 7-15 Years After Delivery AcCording to Waist Circumference (CM)

\begin{tabular}{|c|c|c|c|c|}
\hline Characteristic & $\mathrm{N}$ & Waist $<88 \mathrm{~cm}(\mathrm{~N}=311)$ & Waist $\geq 88 \mathrm{~cm}(\mathrm{~N}=365)$ & $\mathrm{p}$ \\
\hline \multicolumn{5}{|l|}{ POUCH pregnancy (1998-2004) } \\
\hline Gestational age at enrollment, weeks & 676 & $22.5(0.14)$ & $22.2(0.14)$ & 0.135 \\
\hline Gestational age at delivery, weeks & 676 & $39.1(0.10)$ & $39.1(0.10)$ & 0.734 \\
\hline Maternal age at delivery, years & 676 & $27.2(0.37)$ & $26.6(0.35)$ & 0.239 \\
\hline Prior parity & 676 & $0.79(0.07)$ & $1.06(0.08)$ & 0.010 \\
\hline Prepregnancy BMI, $\mathrm{kg} / \mathrm{m}^{2}$ & 676 & $22.5(0.25)$ & $31.4(0.49)$ & $<0.0001$ \\
\hline \multicolumn{4}{|l|}{$\mathrm{GWG}, n(\%)$} & \multirow[t]{4}{*}{0.073} \\
\hline Inadequate & 112 & 47 (11.7) & $65(19.2)$ & \\
\hline Adequate & 124 & $70(23.1)$ & $54(18.7)$ & \\
\hline Excessive & 390 & $170(65.1)$ & $220(62.2)$ & \\
\hline \multicolumn{4}{|l|}{ Hypertensive disorders, $n(\%)$} & \multirow[t]{4}{*}{0.036} \\
\hline None & 599 & $285(93.5)$ & $314(86.4)$ & \\
\hline Preeclampsia or gestational hypertension & 52 & 19 (4.9) & $33(9.4)$ & \\
\hline Chronic hypertension & 25 & $7(1.7)$ & $18(4.2)$ & \\
\hline Gestational diabetes, $n(\%)$ & 40 & $10(3.8)$ & $30(9.3)$ & 0.026 \\
\hline Preterm birth, $n(\%)$ & 169 & $78(10.2)$ & $91(11.2)$ & 0.603 \\
\hline African American, $n(\%)$ & 248 & $92(18.0)$ & $156(30.7)$ & 0.005 \\
\hline \multicolumn{4}{|l|}{ Medicaid status, $n(\%)$} & \multirow{3}{*}{0.0002} \\
\hline On Medicaid & 346 & $136(36.9)$ & $210(53.8)$ & \\
\hline Never on Medicaid & 329 & $174(63.1)$ & $155(46.2)$ & \\
\hline \multicolumn{4}{|l|}{ Education, $n(\%)$} & \multirow{5}{*}{0.001} \\
\hline$<12$ years & 130 & $56(16.3)$ & $74(18.3)$ & \\
\hline HS Diploma & 178 & $69(20.7)$ & 109 (28.6) & \\
\hline Vocational training/Some college & 203 & $81(27.5)$ & $122(34.4)$ & \\
\hline College graduate & 165 & $105(35.5)$ & $60(18.8)$ & \\
\hline \multicolumn{4}{|l|}{ Smoking, $n(\%)$} & \multirow[t]{5}{*}{0.393} \\
\hline Did not smoke during pregnancy & 491 & $233(76.3)$ & $258(69.6)$ & \\
\hline Stopped before enrollment & 67 & $26(9.6)$ & $41(10.9)$ & \\
\hline Smoked $<1 / 2$ pack/day at enrollment & 76 & $32(8.6)$ & 44 (11.4) & \\
\hline Smoked $>1 / 2$ pack/day at enrollment & 42 & $20(5.5)$ & $22(8.1)$ & \\
\hline \multicolumn{5}{|l|}{ POUCHmoms follow-up visit (2010-2014) } \\
\hline Maternal age at follow-up, years & 676 & $38.3(0.4)$ & $37.7(0.4)$ & 0.246 \\
\hline Systolic blood pressure, $\mathrm{mmHg}$ & 676 & $109.7(0.8)$ & $119.3(0.9)$ & $<0.0001$ \\
\hline Diastolic blood pressure, $\mathrm{mmHg}$ & 676 & $70.7(0.7)$ & $79.9(0.6)$ & $<0.0001$ \\
\hline Diet quality score & 675 & $54.9(0.7)$ & $52.1(0.7)$ & 0.004 \\
\hline MAQ: Average activity, hours/week & 676 & $5.0(0.4)$ & $3.4(0.3)$ & 0.002 \\
\hline Breastfeeding, months & 676 & $6.4(0.5)$ & $3.9(0.3)$ & $<0.0001$ \\
\hline BMI, $\mathrm{kg} / \mathrm{m}^{2}$ & 676 & $24.5(0.22)$ & $37.4(0.47)$ & $<0.0001$ \\
\hline \multicolumn{4}{|l|}{ Total parity, $n(\%)$} & \multirow{5}{*}{0.212} \\
\hline 1 & 60 & $22(6.5)$ & $38(9.2)$ & \\
\hline 2 & 259 & $128(43.9)$ & $131(38.9)$ & \\
\hline 3 & 208 & $103(33.0)$ & $105(29.3)$ & \\
\hline $4+$ & 149 & $58(16.6)$ & $91(22.5)$ & \\
\hline \multicolumn{4}{|l|}{ Education level at follow-up, $n(\%)$} & \multirow[t]{5}{*}{0.0001} \\
\hline$<12$ years & 52 & $22(5.5)$ & $30(7.9)$ & \\
\hline HS Diploma & 94 & $31(9.5)$ & $63(15.7)$ & \\
\hline Vocational training/Some college & 339 & $143(45.0)$ & $196(54.7)$ & \\
\hline College graduate & 191 & $115(39.9)$ & $76(21.7)$ & \\
\hline \multicolumn{4}{|l|}{ Cumulative Medicaid status, $n(\%)$} & \multirow[t]{5}{*}{$<0.0001$} \\
\hline Never on Medicaid & 290 & $161(60.3)$ & $129(40.2)$ & \\
\hline Medicaid before POUCH only & 134 & $61(17.3)$ & $73(20.1)$ & \\
\hline Medicaid after POUCH only & 39 & $13(2.8)$ & $26(5.9)$ & \\
\hline Medicaid before, during, and after POUCH & 212 & $75(19.6)$ & $137(33.8)$ & \\
\hline
\end{tabular}

Mean (SE) unless otherwise noted.

Accounting for sampling weights. 
women who breastfed $>6$ months versus $30.7 \%$ of women who breastfed $\leq 6$ months $(p<0.0001)$. Parity before the POUCH pregnancy and gestational diabetes were not associated with breastfeeding duration.

At the POUCHmoms follow-up visit (Table 1), women who breastfed $>6$ months had lower BMI, systolic and diastolic blood pressures, as well as smaller waist and hip circumference compared to women who breastfed $\leq 6$ months. In addition, these women had higher diet quality scores $(p<0.0001)$, had increased hours of physical activity per week $(p=0.018)$, and were more likely to have never smoked $(p<0.0001)$. Overall, lower socioeconomic status during and after pregnancy was inversely associated with breastfeeding duration and total parity did not differ across the groups.

At the follow-up visit, nearly $54.0 \%$ of women exhibited central obesity, defined as $\mathrm{WC} \geq 88 \mathrm{~cm}$ (Table 2). Women with WC $<88 \mathrm{~cm}$ reported having breastfed for 6.4 months, on average, compared to 3.9 months for women with WC $\geq 88 \mathrm{~cm}(p<0.0001)$. Median WC was smallest among women who breastfed $>6$ months (Fig. 2).

In multivariable linear regression analyses, breastfeeding evaluated as a continuous variable was related to smaller WC, such that each additional month of breastfeeding was associated with a $0.20 \mathrm{~cm}$ smaller WC (Table 3). However, this association was attenuated (95\% CI: -0.4 to 0.0 ) in the full model. For women breastfeeding $>6$ months, WC was $4.4 \mathrm{~cm}$ (95\% CI: -7.2 to -1.6$)$ smaller compared to women who did not breastfeed at all and $3.5 \mathrm{~cm}(95 \% \mathrm{CI}:-5.7$ to -1.2$)$ smaller compared to those who breastfed $\leq 6$ months, after full covariate adjustment. Similar regression analyses performed with BMI as a continuous measure of overall adiposity revealed comparable results. No interactions existed between breastfeeding and GWG, smoking history, follow-up time, or total parity in the association with WC or BMI at follow-up (all interactions $p \geq 0.384$ ). Breastfeeding $>6$ months was associated with lower BMI $\left(-1.6 \mathrm{~kg} / \mathrm{m}^{2}, 95 \% \mathrm{CI}\right.$ : -2.6 to -0.6$)$ compared to breastfeeding $\leq 6$ months after adjustment for confounders (Supplementary Table S1).

Individual propensity scores were matched to create a paired sample of $N=330$, while propensity weighting retained the full analytic sample. Propensity weighting minimized absolute standard differences by $\sim 10 \%$ more than matching and appeared to produce adequate balance between preexposure covariates for the two breastfeeding groups, $>6$ months and $\leq 6$ months (Fig. 3). Matched models illustrated a relationship between breastfeeding $>6$ months and WC $(-3.1 \mathrm{~cm}, 95 \% \mathrm{CI}:-5.5$ to -0.7$)$ (Table 4$)$. In addition, propensity weighted models that utilized the full sample estimated that WC was $3.6 \mathrm{~cm}(95 \% \mathrm{CI}$ : -5.6 to -1.6$)$ smaller in women breastfeeding $>6$ months compared to the referent and yielded the smallest standard error of the estimate among all statistical approaches. These results were very similar to the multivariable linear regression model (Table 3).

\section{Discussion}

Our results indicate that women who breastfed more than 6 months after the POUCH Study pregnancy had less central adiposity $7-15$ years later compared to women who breastfed 6 months or less, accounting for confounding by age, race/ ethnicity, parity and pregnancy complications, socioeconomic status, and healthy lifestyle factors. While we detected a modest linear dose-response relationship between months of breastfeeding and $\mathrm{WC}$, our results raise the possibility that a threshold effect may exist, such that breastfeeding for more than 6 months is associated with improved long-term maternal central adiposity. Importantly, these findings were robust across multivariable linear regression models as well as propensity score analyses to indicate that the association of breastfeeding duration and WC may be adequately estimated regardless of the analytic method used. Our results suggest that despite systematic differences in overall healthier lifestyles between women who do and do not breastfeed, those who breastfeed for more than 6 months have smaller WC on average. In addition, while the results for both the WC and BMI models validate one another, we emphasize WC as the outcome of interest given that central adiposity is a better predictor of long-term cardiometabolic and cardiovascular disease risk than BMI alone.

Our findings are in agreement with several previous studies that report an association between breastfeeding exposure and central adiposity. Studies with shorter follow-up periods ( $<3$ years) reported greater overall weight loss for women
FIG. 2. Comparative waist circumference (cm) according to breastfeeding duration. Line indicates central obesity cutoff point for women at $88 \mathrm{~cm}$.

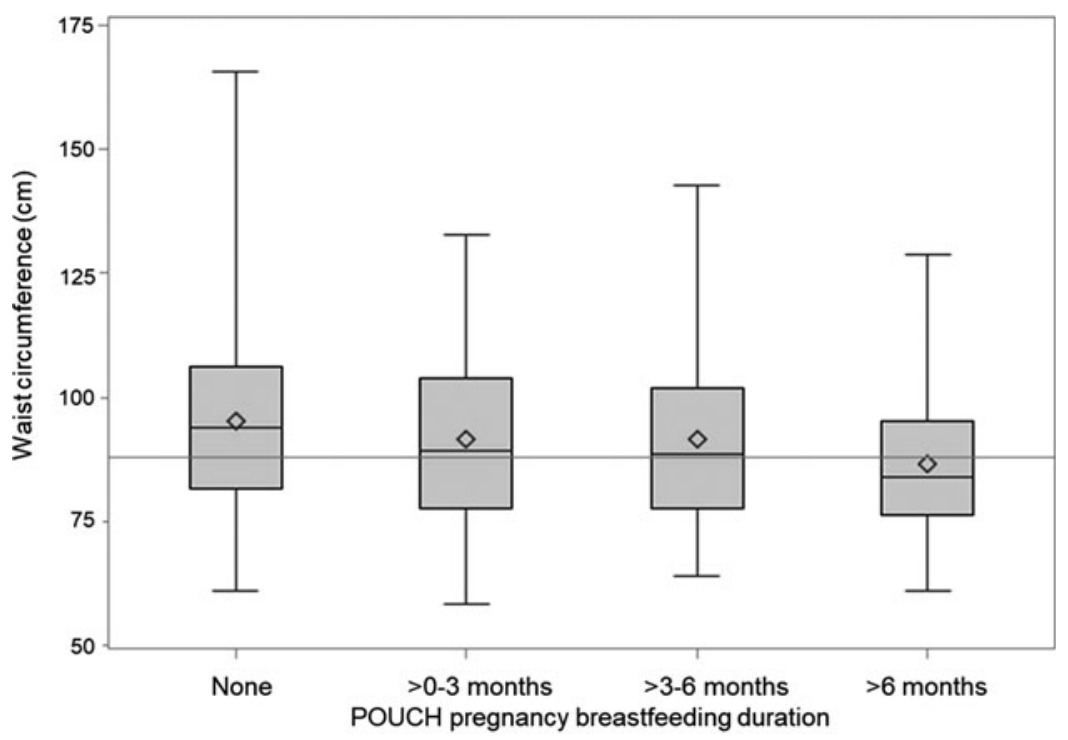




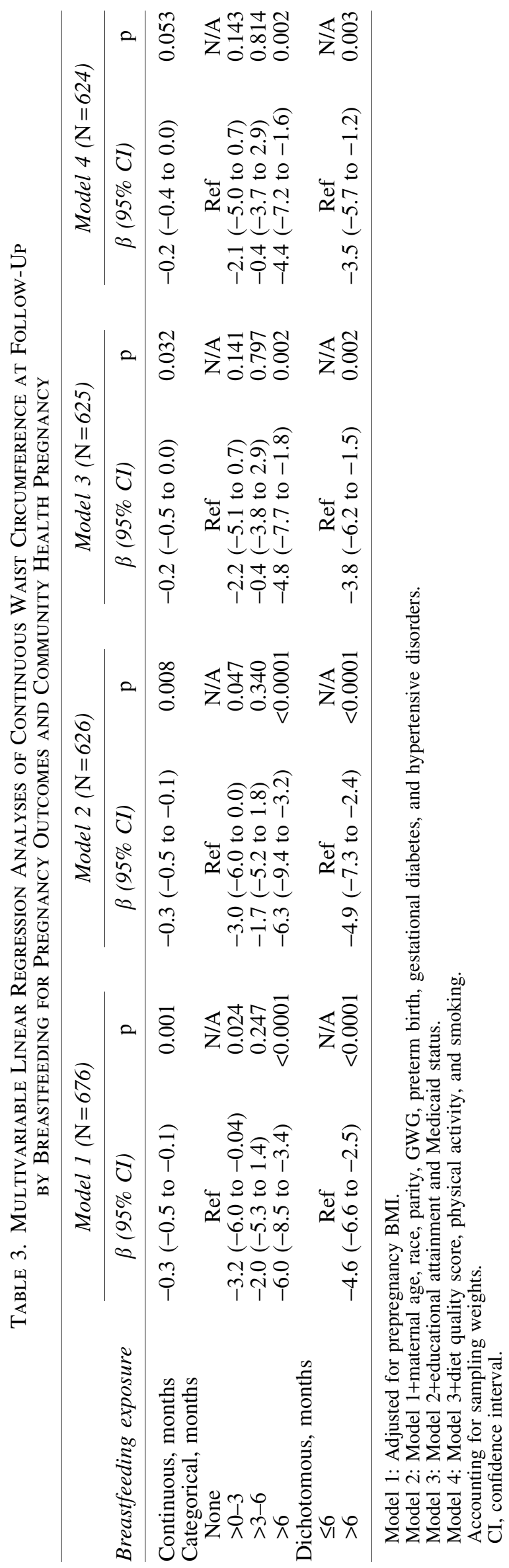

who breastfed for 6 months, with no apparent linear doseresponse relationship. ${ }^{12,14}$ However, a systematic review of weight loss or change in body composition up to 2 years postpartum found that a majority of studies failed to detect any association with breastfeeding duration. ${ }^{18}$ Alternatively, longer observational periods ( $>7$ years) may offer more definitive results regarding this relationship.

A path analysis of the Danish National Birth Cohort found that any breastfeeding was directly related to lower maternal WC adjusted for BMI 7 years later. ${ }^{13}$ McClure et al. evaluated the effect of lifetime breastfeeding duration for cumulative births and reported comparable findings in two separate analyses. At the WISH (Women and Infant Study of Healthy Hearts) 7-year follow-up visit, women who breastfed following each singleton pregnancy for 3 months or less had more abdominal visceral fat deposits, measured by computed tomography, compared to women who breastfed $>3$ months. ${ }^{31}$ Similarly, in the SWAN (Study of Women's Health Across the Nation), women who breastfed each child for at least 3 months had smaller WC as well as less subcutaneous and visceral adiposity $17-22$ years postpartum than women who did not consistently breastfeed all children, suggesting that breastfeeding may mobilize abdominal fat. ${ }^{17}$ Although these results collectively suggest that a relationship exists between longer breastfeeding duration and lower longterm maternal central adiposity, our study did not detect differences in the outcome measures and breastfeeding $>3$ months following a single pregnancy. Consequently, variation in the definition of breastfeeding duration across one or more pregnancies continues to make direct comparisons between this study and the existing literature difficult.

It is well established that BMI does not distinguish among areas of fat distribution and pregnancy contributes to the accumulation of abdominal adiposity. ${ }^{6,8-10}$ One Swedish study reported that a $68 \%$ increase in adipose tissue during pregnancy occurred within the trunk region. ${ }^{32}$ Currently, there is additional emphasis to consider the role of prepregnancy fat distribution, beyond BMI, and its relationship to health outcomes. Prepregnancy abdominal adiposity has been independently associated with overall and central obesity, GWG, and breastfeeding behavior. ${ }^{13,33}$ Kirkegaard et al. showed that prepregnancy WC and BMI, mutually adjusted for one another, were differentially associated with GWG and odds of ever breastfeeding. ${ }^{33}$ Therefore, controlling for prepregnancy WC versus BMI may result in incongruent associations between breastfeeding and long-term maternal adiposity.

Several limitations of this study deserve mention. Prepregnancy WC was not measured and could not be controlled for in regression models. Although BMI was highly correlated with WC at follow-up ( $\rho=0.939, p<0.0001)$, we cannot be sure that prepregnancy BMI is an acceptable proxy for prepregnancy WC. Consequently, we were unable to disentangle overall adiposity from central adiposity. Imprecise recall of breastfeeding duration, particularly among women who reported breastfeeding for less than 6 months, may have resulted in misclassification of the exposure variable. Maternal recall up to 20 years postpartum has been validated when breastfeeding is culturally commonplace and duration is long. ${ }^{34}$ Yet, although breastfeeding initiation has increased over the past several years in the United States, rates for continued duration remain low. ${ }^{35}$ Therefore, it is plausible that women who reported breastfeeding less than 6 months had 


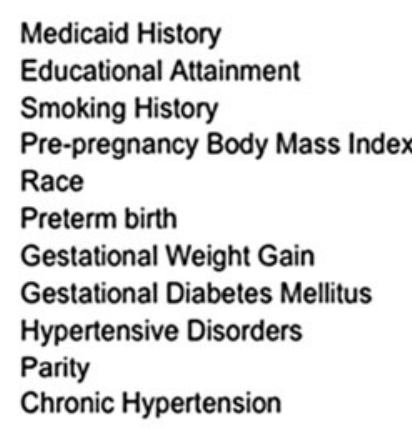

FIG. 3. Group balance for breastfeeding before and after applying propensity score matching and weighting methods.
Absolute Standardized Difference - Propensity Matched

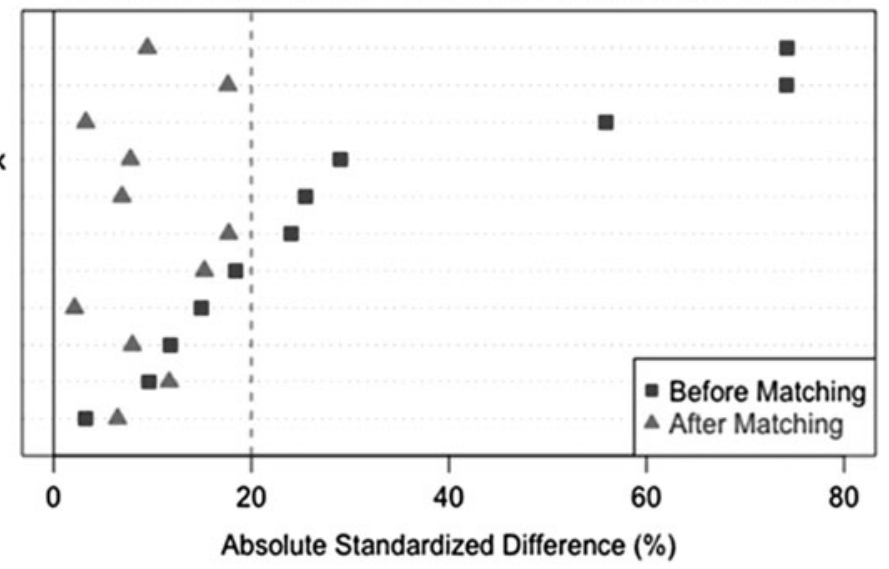

\section{Absolute Standardized Difference - Propensity Weighted}

Medicaid History
Educational Attainment
Smoking History
Pre-pregnancy Body Mass Index
Race
Preterm birth
Gestational Weight Gain
Gestational Diabetes Mellitus
Hypertensive Disorders
Parity
Chronic Hypertension

poor recall and may have been misclassified in the $>0-3$ and $>3-6$ month breastfeeding groups in our analysis. By combining these groups and looking at breastfeeding duration as a dichotomous variable, we were able to reduce misclassification bias. In addition, our breastfeeding data did not capture breastfeeding consistency or intensity, nor did it include specific information on breast milk expression by pumping or workplace policies, which may play a large role in whether mothers are able to continue breastfeeding for longer durations. ${ }^{36}$

This analysis focused solely on breastfeeding duration for the POUCH Study pregnancy to conduct propensity score approaches, which required detailed clinical data before and during pregnancy. Future analyses should account for lifetime breastfeeding duration to more fully understand the cumulative influence on maternal central adiposity. In addition, nearly half of the eligible women who participated in the POUCH Study subcohort did not attend the POUCHmoms follow-up visit and were notably different from those who did, possibly introducing selection bias. Finally, propensity score methods assume that all factors affecting treatment assignment and outcome have been measured, and we recognize that there are unmeasured factors, such as prepregnancy diet and exercise, which we were unable to account for. ${ }^{21}$

Our study uniquely incorporates two propensity score approaches to account for potential bias due to overall healthy lifestyle on the likelihood of breastfeeding. Previous obser- vational studies reported that predominantly white women of higher socioeconomic status, who made healthier diet and exercise choices, were more likely to breastfeed and have better overall health. ${ }^{18,19}$ Previous randomized controlled trials have assessed effects of breastfeeding promotion interventions on breastfeeding duration, but not on long-term maternal health outcomes. ${ }^{37,38}$ Our novel application of propensity score methods was appropriate to simulate a randomized study design and minimize potential bias by creating balanced groups conditioned on prepregnancy and delivery covariates. By balancing baseline covariates, more valid inferences could be made about the treatment effect of breastfeeding on WC. ${ }^{39}$ Importantly, not all propensity score approaches may appropriately estimate the treatment effect better than traditional regression covariate adjustment. We have attempted to mitigate this concern by evaluating more than one propensity score method. ${ }^{40}$ Our analyses show that estimates from both propensity score matching and weighting were comparable and corroborated the results from the multivariable linear regression models.

In conclusion, breastfeeding greater than 6 months was associated with smaller maternal WC, accounting for age, race/ethnicity, parity, pregnancy complications, socioeconomic status, and lifestyle factors, compared to breastfeeding less than or equal to 6 months. Using multivariable linear regression modeling as well as propensity score approaches 


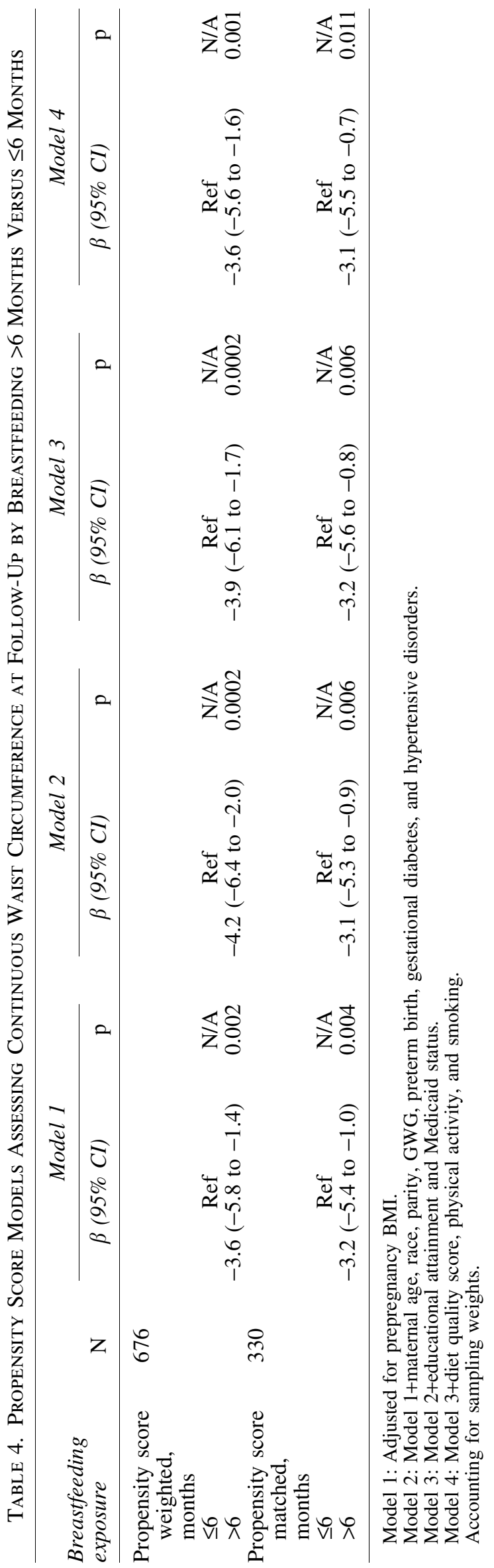

to test the same exposure-outcome association, we consistently detected that a threshold effect may exist for breastfeeding greater than 6 months. Our results warrant further analyses of cumulative lifetime duration of breastfeeding to understand the magnitude of the relationship with maternal central adiposity over time. Breastfeeding duration may be important to consider when studying long-term maternal cardiovascular and metabolic health.

\section{Acknowledgments}

POUCHmoms Study was supported by the National Heart, Blood, and Lung Institute (R01-HL103825). The parent POUCH Study was supported by the National Institute of Child Health and Human Development and the National Institute of Nursing Research (R01 HD034543), the March of Dimes Foundation Perinatal Epidemiological Research Initiative Program (20-FY98-0697 through 20-FY04-37), the Thrasher Research Foundation (02816-7), and the Centers for Disease Control and Prevention (U01 DP000143-01).

\section{Authors' Contributions}

Substantive contributions have been made by all authors and their written permission has been obtained. C.H. and J.M.C. designed the study; B.B., J.M.C., and G.G.S. acquired the data; M.B. supervised the statistical analysis; G.G.S. and T.S. analyzed the data; G.G.S. wrote the article; and all authors contributed to the interpretation of the results and read, approved the final version of the article, and agreed with its content.

\section{Author Disclosure Statement}

No competing financial interests exist.

\section{References}

1. Fifty-fifth World Health Assembly. Infant and young child nutrition. Geneva, Switzerland: World Health Organization, 2002 (WHA55.25). Available at: http://apps.who.int/ gb/archive/pdf_files/WHA55/ea5515.pdf?ua=1 Accessed July 1, 2016.

2. Chowdhury R, Sinha B, Sankar MJ, et al. Breastfeeding and maternal health outcomes: A systematic review and metaanalysis. Acta Paediatr 2015;104:96-113.

3. Gunderson EP. Childbearing and obesity in women: Weight before, during, and after pregnancy. Obstet Gynecol Clin North Am 2009;36:317-ix.

4. Fowler-Brown AG, De Boer IH, Catov, JM, et al. Parity and the association with diabetes in older women. Diabetes Care 2010;33:1778-1782.

5. Gunderson EP, Lewis CE, Murtaugh MA, Quesenberry CP, West DS, Sidney S. Long-term plasma lipid changes associated with a first birth: The coronary artery risk development in young adults study. Am J Epidemiol 2004; 159:1028-1039.

6. Gunderson EP, Sternfeld B, Wellons MF, et al. Childbearing may increase visceral adipose tissue independent of overall increase in body fat. Obesity 2008;16:1078-1084.

7. Prentice AM, Jebb SA. Beyond body mass index. Obes Rev 2001;2:141-147.

8. Klein S, Allison DB, Heymsfield SB, et al. Waist circumference and cardiometabolic risk: A consensus statement from Shaping America's Health: Association for Weight Management and Obesity Prevention; NAASO, the Obesity Society; 
the American Society for Nutrition; and the American Diabetes Association. Am J Clin Nutr 2007;85:1197-1202.

9. Coutinho T, Goel K, Corrêa de Sá D, et al. Combining body mass index with measures of central obesity in the assessment of mortality in subjects with coronary disease. J Am Coll Cardiol 2013;61:553-560.

10. Janssen I, Katzmarzyk PT, Ross R. Waist circumference and not body mass index explains obesity-related health risk. Am J Clin Nutr 2004;79:379-384.

11. Butte NF, Wong WW, Hopkinson JM. Energy requirements of lactation women derived from doubly labeled water and milk energy output. J Nutr 2001;131:53-58.

12. Baker JL, Gamborg M, Heitmann BL, Lissner L, Sørensen TIA, Rasmussen KM. Breastfeeding reduces postpartum weight retention. Am J Clin Nutr 2008;88:1543-1551.

13. Kirkegaard H, Stovring H, Rasmussen KM, Abrams B, Sørensen TIA, Nohr EA. How do pregnancy-related weight changes and breastfeeding relate to maternal weight and BMI-adjusted waist circumference 7 y after delivery? Results from a path analysis. Am J Clin Nutr 2014;99:312-319.

14. Dewey KG, Heinig MJ, Nommsen LA. Maternal weightloss patterns during prolonged lactation. Am J Clin Nutr 1993;58:162-166.

15. Jarlenski MP, Bennett WL, Bleich SN, Barry CL, Stuart EA. Effects of breastfeeding on postpartum weight loss among U.S. women. Prev Med 2014;69:146-150.

16. Sharma AJ, Dee DL, Harden SM. Adherence to breastfeeding guidelines and maternal weight 6 years after delivery. Pediatrics 2014;134(Suppl 1):S42-S49.

17. McClure CK, Schwarz EB, Conroy MB, Tepper PG, Jannsen I, Sutton-Tyrrell KC. Breastfeeding and subsequent maternal visceral adiposity. Obesity 2011;19:2205.

18. Neville CE, McKinley MC, Holmes VA, Spence D, Woodside JV. The relationship between breastfeeding and postpartum weight change-A systematic review and critical evaluation. Int J Obes (Lond) 2014;38:577-590.

19. Stuebe AM, Rich-Edwards JW. The reset hypothesis: Lactation and maternal metabolism. Am J Perinatol 2009; 26:81-88.

20. Pesa JA, Shelton MM. Health-enhancing behaviors correlated with breastfeeding among a national sample of mothers. Public Health Nurs 1999;16:120-124.

21. Austin PC. An introduction to propensity score methods for reducing the effects of confounding in observational studies. Multivariate Behav Res 2011;46:399-424.

22. Austin PC. Balance diagnostics for comparing the distribution of baseline covariates between treatment groups in propensityscore matched samples. Stat Med 2009;28:3083-3107.

23. Rubin DB. The design versus the analysis of observational studies for causal effects: Parallels with the design of randomized trials. Stat Med 2007;26:20-36.

24. Holzman C, Bullen B, Fisher R, Paneth N, Reuss L; the Prematurity Study Group. Pregnancy outcomes and community health: The POUCH study of preterm delivery. Paediatr Perinat Epidemiol 2001;15(Suppl 2):136-158.

25. Lean MEJ, Han TS, Morrison CE. Waist circumference as a measure for indicating needs for weight management. BMJ 1995;311:158-161.
26. American College of Obstetricians and Gynecologists Weight gain during pregnancy. Committee Opinion No. 548. Obstet Gynecol 2013;121:210-212.

27. Roberts JM, August PA, Bakris G, et al. Hypertension in pregnancy: Report of the American College of Obstetricians and Gynecologists' Task Force on Hypertension in Pregnancy. Obstet Gynecol 2013;122:1122-1131.

28. Chiuve SE, Fung TT, Rimm EB, et al. Alternative dietary indices both strongly predict risk of chronic disease. J Nutr 2012;5:1009-1018.

29. Vuillemin A, Oppert J, Guillemin F, et al. Self-administered questionnaire compared with interview to assess past-year physical activity. Med Sci Sports Exerc 2000;32:1119-1124.

30. Rosenbaum PR, Rubin DB. The central role of the propensity score in observational studies for causal effects. Biometrika 1983;70:41-50.

31. McClure CK, Catov J, Ness R, Schwarz EB. Maternal visceral adiposity by consistency of lactation. Matern Child Health J 2012;169:10.

32. Sohlström A, Forsum E. Changes in adipose tissue volume and distribution during reproduction in Swedish women as assessed by magnetic resonance imaging. Am J Clin Nutr 1995;61:287-295.

33. Kirkegaard H, Nohr EA, Rasmussen KM, et al. Maternal prepregnancy waist circumference and $\mathrm{BMI}$ in relation to gestational weight gain and breastfeeding behavior: The CARDIA study. Am J Clin Nutr 2015;102:393-401.

34. Natland ST, Andersen LF, Nilsen TI, Forsmo S, Jacobsen GW. Maternal recall of breastfeeding duration twenty years after delivery. BMC Med Res Methodol 2012;12:179.

35. National Center for Chronic Disease Prevention and Health Promotion. Breastfeeding Report Card 2016. Available at: www.cdc.gov/breastfeeding/pdf/2016breastfeedingreport card.pdf Accessed November 2, 2017.

36. Murtagh L, Moulton AD. Working mothers, breastfeeding, and the law. Am J Public Health 2011;101:217-223.

37. Kramer MS, Chalmers B, Hodnett ED, et al. Promotion of breastfeeding intervention trial (PROBIT): A randomized trial in the Republic of Belarus. JAMA 2001;285:413-420.

38. Kim SK, Park S, Oh J, Kim J, Ahn S. Interventions promoting exclusive breastfeeding up to six months after birth: A systematic review and meta-analysis of randomized controlled trials. Int J Nurs Stud 2018;80:94-105.

39. D'Agostino RB. Propensity scores in cardiovascular research. Circulation 2007;115:2340-2343.

40. Elze MC, Gregson J, Baber U, et al. Comparison of propensity score methods and covariate adjustment: Evaluation in 4 cardiovascular studies. J Am Coll Cardiol 2017;69:345-357.

Address correspondence to:

Gabrielle G. Snyder, MPH

Department of Epidemiology

Graduate School of Public Health University of Pittsburgh

130 De Soto Street Pittsburgh, PA 15261

E-mail: gas54@pitt.edu 